\title{
Martha Nussbaum über das Phänomen der Angst: Eine kritische Relektüre
}

\author{
Eva Bobst/Angela Kallhoff/Anna Wieder
}

Wir alle kennen dieses Gefühl der lähmenden Beklemmung und der vermeintlichen Ausweglosigkeit, das manchmal in Wut oder Zorn umschlägt. Denn die Angst begleitet uns, seit wir denken können. Als Kind fürchten wir uns vor der Dunkelheit im Keller und vor dem knarrenden Holzboden in der Nacht. Als Erwachsene fürchten wir uns vor (geistigen und geographischen) Abgründen, vor Spinnen, Flügen und Krankheiten. Wir fürchten uns aber auch vor Kriegen, dem Klimawandel, vor politisch und ökonomisch unsicheren Zeiten sowie ganz allgemein vor Dingen, die wir nicht verstehen. Wir fürchten nicht nur um uns selbst, sondern auch um andere Menschen, ja, sogar um den Planeten Erde können wir fürchten. Wir fürchten uns manchmal vor unserer Zukunft, und manchmal fürchten wir um unsere Zukunft, wie insbesondere die KlimaaktivistInnen der Fridays-for-Future-Bewegung aktuell in Erinnerung rufen.

In der politischen Philosophie werden die Angst und Politiken der Angst zunehmend als Bedrohungen für das Verfahren demokratischer Meinungsbildung und eine kritische demokratische Öffentlichkeit diskutiert. ${ }^{1}$ Denn sowohl die Angst als auch ihre Kehrseite, das gesteigerte Sicherheitsbedürfnis, lassen die Überzeugungskraft demokratischer Grundwerte wie Freiheit, Pluralität und kollektiver Selbstbestimmung immer stärker erodieren. Auch die US-amerikanische Moralphilosophin Martha Nussbaum identifiziert in ihrem jüngst vorgelegten Buch Königreich der Angst. Gedanken zur aktuellen politischen Krise ${ }^{2}$ die Angst als Gefahr für die Demokratie. Denn die politisch motivierte Angst, so Nussbaum, stehe durch ihre egoistische und ausgrenzende Qualität jeder demokratischen Gemeinsamkeit notwendigerweise im Weg. Infolgedessen müssen wir in der Lage sein, die Angst zu überwinden, wenn wir das demokratische

\footnotetext{
Vgl. u.a. Brian Massumi (Hg.), The Politics of Everyday Fear, Minneapolis/London 1993; Wendy Brown, Mauern. Die neue Abschottung und der Niedergang der Souveränität [2010/2017], Frankfurt am Main 2018; Ruth Wodak, The Politics of Fear. What Right-Wing Populist Discourses Mean, London 2015; Bernd Greiner, United States of Angst. Donald Trump und der Extremismus der Mitte, in: Blätter für deutsche und internationale Politik 61/9 (2016), 43-50.

2 Martha C. Nussbaum, Königreich der Angst. Gedanken zur aktuellen politischen Krise [2018], Darmstadt 2019.
} 
Miteinander fördern und erhalten wollen. Nussbaums Lösungsvorschlag ist auf den ersten Blick denkbar einfach: Wenn es uns gelingt, anstelle von Angst Hoffnung zu empfinden, lassen sich schwierige Situationen, in denen die Angst eine tragende Rolle spielt, in einem demokratisch wertvollen Sinne positiv verändern.

In diesem Beitrag möchten wir ausgehend von Nussbaums Überlegungen zu Emotionen eine Diskussion über das Phänomen der Angst führen. Die Untersuchungen in diesem Beitrag sind dabei von drei Thesen geleitet: Erstens ist Nussbaums jüngster Beitrag zur Debatte um Angst nur dann in einer gelingenden Weise zu würdigen, wenn auch ihre grundlegenden Einsichten zum umfassenderen Phänomen der Emotionen zur Kenntnis genommen werden. Entsprechend wird in diesem Beitrag eine Kontextualisierung von Nussbaums Theorie der Angst im Rahmen ihrer allgemeinen Theorie der Emotionen unternommen. Zweitens muss eine auch politisch gedachte Auseinandersetzung mit dem Phänomen der Angst als ein wichtiger Horizont der Ausdeutung verstanden werden. Wir werden diesen Anspruch ernst nehmen, indem wir den Horizont weiten und nach Angst als einem Phänomen in der heutigen politischen Theoriebildung fragen. Vor diesem Hintergrund soll Nussbaum drittens nicht nur affirmierend gelesen werden. Nussbaum ist eine Denkerin, die bewusst und gewollt Provokationen setzt und Debatten anregt. Wir werden diese Provokation aufnehmen und sie auf eine konkrete und aktuelle politische Auseinandersetzung beziehen: Die Fridays-for-Future-Bewegung und die in dieser Bewegung so zentrale Person Greta Thunberg dienen uns als ein Anwendungsbeispiel, an dem zugleich die Grenzen der Nussbaum'schen Theoriebildung diskutiert werden können.

Unsere Ausführungen gliedern sich in vier Abschnitte. Im ersten Abschnitt wird mit einer Skizze der Nussbaum'schen Emotionentheorie und deren Verortung in ihrer politischen Theorie ein Rahmen abgesteckt, in welchem auch die Ausführungen zur Angst verhandelt werden können. Im zweiten Abschnitt wird Nussbaums Angstkonzeption näher beleuchtet und erläutert, inwiefern ihre Kontextualisierung von Emotionen und insbesondere von Angst einen Grundton der aktuellen politischen Debatte trifft. Eine besondere Deutlichkeit gewinnt die politische Dimension der Angst in Auseinandersetzung mit den Statements der Vorreiterin der Fridaysfor-Future-Bewegung, Greta Thunberg, die im dritten Abschnitt zu Wort kommt. Ausgehend von Thunbergs Bezugnahme auf die Emotion der Angst unterziehen wir Nussbaums Konzeption einer kritischen Lektüre. Dabei zeigen wir, dass Nussbaum zwar zentrale 
Charakteristika der Angst zu benennen weiß, anderen positiven Funktionen und Potentialen, die die Emotion der Angst im Politischen zu entfalten vermag, aber nicht genug Beachtung schenkt. Der vierte Abschnitt schließt mit einer resümierenden Einordnung von Nussbaums Angstkonzeption, auf Basis derer wir einen Ausblick auf das noch zu schöpfende Potential von Nussbaums Überlegungen geben.

\section{Nussbaums Theorie der Emotionen}

Martha Nussbaum hat sich in den letzten Jahren verstärkt mit der Rolle von Emotionen im Politischen auseinandergesetzt. Ihre Überlegungen zeichnen sich dabei durch das Ziel aus, ethische Theorien der Antike für Fragen der internationalen sozialen Gerechtigkeit fruchtbar zu machen und damit in der aktuellen Debatte einen Gegenpol zu bilden. Dieses Anliegen verfolgt Nussbaum auch in ihren jüngsten Auseinandersetzungen mit der Emotion der Angst. In ihrem Buch Königreich der Angst unternimmt sie nicht nur eine umfassende Analyse dieser Emotion, sondern verfolgt auch ein politisch-theoretisches Anliegen: Sie möchte die Angst der Manipulation durch Andere entziehen. Um ihre Beiträge im Kontext ihrer Theorie der politischen Emotionen genauer zu verorten, soll zunächst deren umfassender theoretische Hintergrund erläutert werden.

Nussbaum hat sich nicht erst jetzt, sondern schon in Hiding from Humanity: Disgust, Shame, and the Law ${ }^{3}$ und in Upheavals of Thought. The Intelligence of Emotions ${ }^{4}$ intensiv mit dem Thema der Emotionen befasst. Auch wenn dieses Thema in der praktischen Philosophie inzwischen ein breites Forschungsfeld geworden ist, ${ }^{5}$ konnte Nussbaum nicht nur eigene Akzente setzen, sondern hat das Feld der Untersuchung auch abgesteckt. Die Gefühle, denen sie sich in ihrem Werk widmet, umfassen so unterschiedliche Phänomene wie Fürsorge, Scham und Ekel, Mitleid und Liebe, sowie neuerlich Wut und Angst. ${ }^{6}$ Es kann nicht Ziel der Ausführungen sein, die Angst in

\footnotetext{
3 Martha C. Nussbaum, Hiding from Humanity: Disgust, Shame and the Law, Princeton 2004.

4 Dies., Upheavals of Thought. The Intelligence of Emotions, Cambridge ${ }^{8} 2008$.

5 Vgl. Sabine A. Döring, Philosophie der Gefühle, Frankfurt am Main 2009.

${ }^{6}$ Martha C. Nussbaum, Konstruktion der Liebe, des Begehrens, und der Fürsorge: drei philosophische Aufsätze [2002], Stuttgart 2012; Dies., Hiding from Humanity; Dies., Political Emotions: Why Love Matters for Justice, Cambridge 2008; Dies., Königreich der Angst.
} 
dieses breite Spektrum von Gefühlen einzuordnen - dies wäre auch angesichts der Verschiedenartigkeit der von Nussbaum erörterten Phänomene nicht möglich. Stattdessen sollen einige Besonderheiten der Nussbaum'schen Theorie der Emotionen erläutert werden. Nur vor diesem Hintergrund ist es auch möglich, das komplexe Phänomen der Angst zu entschlüsseln.

In der Theorie der Emotionen ist es eine Grundlagenfrage, ob Emotionen wie Liebe oder Hass rational zugänglich und gestaltbar sind oder ob sie als Affekte einer überlegten Gestaltung unzugänglich bleiben. Eine typische Antwort auf die Frage, wie das Verhältnis von Rationalität und Gefühl zu denken ist, besteht darin, zwischen unterschiedlichen Typen von Gefühlen zu unterscheiden. Während Ekel oder Wut pure Gefühle sind, können Liebe und Fürsorge als Emotionen gedeutet werden, die einer überlegten Gestaltung zugänglich sind. Entgegen dieser auf den ersten Blick plausibel scheinenden Einteilung verteidigt Nussbaum die These, dass jedes Gefühl nicht nur rational zugänglich ist, sondern einen kognitiven Anteil hat. Auch wenn Emotionen nicht im strikten Sinn rational sind - sie können nicht wahr oder falsch sein -, haben sie "reasonableness «? Und dies rührt daher, dass sie notwendigerweise Überzengungen beinhalten. "Another important point, much stressed by Aristotle and other philosophers who write on this topic, is that the beliefs are connected to the emotions in a very intimate way: they appear to be part of what the emotion itself is. $\|^{8}$ Nussbaum geht in diesem Punkt aber über Aristoteles hinaus, indem sie argumentiert, dass Überzeugungen (nebst Gefühlen ("feelings"), die unreflektiert sind und unmittelbar mit körperlichen Reaktionen einhergehen) nicht nur Teil der Emotion sind, sondern diese auch definieren.

Nussbaum entwickelt diesen Aspekt ihrer Emotionentheorie mit Bezug auf die aristotelische Theorie der Emotionen in der Rhetorik, wo Aristoteles das Abhängigkeitsverhältnis zwischen Emotionen und Überzeugungen wie folgt illustriert: Um Emotionen beim Auditorium hervorzurufen, konfrontiert der Sprecher die Zuhörer mit Überzeugungen: Wenn etwa Ärger auf die Perser evoziert wird, bringt der Sprecher Beispiele dafür, dass die Perser einen Schaden mit Rücksicht auf das Wohl der Bevölkerung angerichtet haben. Emotionen basieren also auf Überzeugungen - ja mehr noch, bestimmten Emotionen kann eine distinkte Gruppe von Überzeugungen zugeordnet werden. ${ }^{9}$ Diese Theorie wird auch als eine »kogni-

\footnotetext{
7 Vgl. Nussbaum, Hiding from Humanity, 25.

8 Ebd., 27.

9 Vgl. ebd., 26f.
} 
tivistische Emotionentheorie» bezeichnet. Ergänzend hierzu muss noch angemerkt werden, dass anders als in ihrem Buch Upheavals of Thought später zunehmend davon abgesehen wird, strikt von Urteilen oder intentionalen Objektbezügen bei Emotionen auszugehen. Stattdessen werden "Gedanken« als hinreichend betrachtet für die kognitive Komponente. ${ }^{10}$

Die Ausdeutung von Emotionen mit Rücksicht auf ihren Gehalt als Überzeugungen ist nicht nur ungewöhnlich, sondern hat auch eine unmittelbare Konsequenz für eine weitere wichtige Eigenschaft von Emotionen. Emotionen sind moralisch nicht neutral; vielmehr verdienen sie entweder moralische Anerkennung (»appraisal«), oder sie sind moralisch zu kritisieren und zu verwerfen. ${ }^{11}$ Grundlage für eine kritische Auseinandersetzung mit dem Wert von Emotionen ist allerdings nicht die Gefühls-Seite, sondern die Urteils-Seite. Ich kann zum Beispiel wütend sein auf Peter, weil er mir vermeintlich etwas angetan hat - und es kann sein, dass dies eine falsche Annahme ist. Vor allem gibt es auch den Fall, dass die Tatsachenurteile richtig sind, die daraus erwachsene Überzeugung aber aus einem fehlerhaften Prozess des Schließens resultiert. So kann eine Person eine wertende Sicht vertreten, die zwar wahr ist, aber unvernünftig. Dann resultiert die Einstellung aus einer unachtsamen Weise des Schließens und Nachdenkens. ${ }^{12}$ Wird eine Emotion kritisiert, richtet sich die Kritik insbesondere darauf, dass die eine Emotion bestimmenden Überzeugungen unvernünftig sind, ja möglicherweise aus ungenauem und unachtsamen Nachdenken resultieren. Die Person hätte es besser wissen können, vielleicht sogar sollen - und dann hätte sie auch andere Emotionen.

Eine zweite wichtige Konsequenz aus dem Gesagten ist die Annahme der gezielten Gestaltbarkeit von Emotionen. Es ist unserer Tage nachgerade eine Standardsicht zu Gefühlen, dass sie den Menschen überfallen, und dass man sie zwar hat, aber nicht beeinflussen kann. Menschen werden als Opfer von Gefühlen gedacht - dies gilt in besonderer Weise auch für das Gefühl der Angst. Nussbaum vertritt dagegen in all ihren Ausführungen zu Emotionen nicht nur die Ansicht, dass Personen ihre eigenen Emotionen gestalten können, sondern auch eine These der Erziehbarkeit von Emotionen und der Gestaltbarkeit im politischen Raum. Im Werk Political Emotions. Why Love Matters for Justice wird als eine für den Raum des Politischen besonders wichtige Haltung die "Compassion«, etwas unzulänglich

\footnotetext{
${ }^{10}$ Vgl. Nussbaum, Political Emotions, 399-401.

${ }^{11}$ Vgl. Nussbaum, Hiding from Humanity, $33 \mathrm{f}$.

${ }^{12}$ Vgl. ebd., 33.
} 
mit `Mitgefühl übersetzt, diskutiert. Nussbaum versteht darunter eine schmerzhafte Emotion, die auf ein ernstes Leiden einer anderen Kreatur gerichtet ist. Ihrer kognitivistischen Theorie entsprechend analysiert Nussbaum zunächst die Gedanken, die Mitgefühl konstituieren. Sie beinhalten nicht nur eine Überzeugung betreffend der Ernsthaftigkeit des Leidens und des Nicht-Verschuldetseins des Leids, sondern auch die Einsicht, in eine vergleichbare Situation geraten zu können, und die Einsicht, dass Glück eine wichtige Konstante ist. ${ }^{13}$ Insbesondere kann ausgehend von einer genauen Definition des Wohlwollens eine Topographie der Gefühlslandschaft entwickelt werden: Angst, Neid und Scham sind Feinde des Wohlwollens, ${ }^{14}$ Liebe dagegen engster Verbündeter. ${ }^{15}$ Vor allem sind Gefühle nicht nur genuiner Bestandteil menschlichen Lebens, sondern auch des politischen Raums. Nussbaum versteht ihre Emotionentheorie auch als eine fundamentale Kritik am politischen Liberalismus. Jener übersieht, dass der politische Raum immer schon emotional durchwirkt ist. Insbesondere verspielt er damit auch jenes wichtige Gestaltungspotential, das Emotionen stets zulassen.

Zusammenfassend entwickelt Nussbaum eine eigenständige und mit anderen Ansätzen nicht vergleichbare Theorie der Emotion. Hervorstechendstes Merkmal ist die Konzentration auf Überzeugungen und Gedanken, welche Emotionen nicht nur bestimmen, sondern sie auch identifizierbar machen. Emotionen sind in dieser Konzeption nicht moralisch neutral, sondern entweder abzulehnen oder zu begrüßen. Und sie sind genuiner Bestandteil sowohl des Lebens der Menschen in der Sphäre des Politischen, wie auch der Politik selbst. Ausgehend von dieser Skizze der Theorie der Emotion wenden wir uns nun Nussbaums Analysen zum Phänomen der Angst zu. Es wird eine überaus spannende Frage sein, ob Nussbaum in ihrer Theorie der Angst ihre vorgeschlagenen Grundideen durchhalten kann: Angst ist auf den ersten Blick gerade kein intellektuelles Phänomen, sondern hat eine dumpfe, überraschende und auch infantile Qualität. Inwiefern Angst dennoch rational gefasst und gestaltet werden kann und ob ihr dadurch möglicherweise eine berechtigte Rolle in der politischen Gestaltung unseres Zusammenlebens zukommt, wird Thema der nächsten zwei Abschnitte sein.

${ }_{13}$ Vgl. Nussbaum, Political Emotions, 142-44.

14 Vgl. ebd., 314-77.

15 Vgl. ebd., $378 f f$. 


\section{Nussbaums Angstkonzeption}

Nussbaums Theorie der Angst basiert, analog ihrer Theorie der Emotionen, im Wesentlichen auf antiken Angstkonzeptionen - bereits Aristoteles hat erkannt, dass sich die Angst meistens auf zukünftige Ereignisse richtet und mit einem Gefühl der Machtlosigkeit einhergeht, dieses Ereignis abzuwehren. ${ }^{16} \mathrm{Um}$ ein hinreichendes Bild der Angst zeichnen zu können, müssten aber, so Nussbaum, auch zeitgenössisch-empirische Forschungsergebnisse berücksichtigt werden. So räumt beispielsweise die psychoanalytische Forschung der Angst eine genetische, chronologische und kausale Vorrangstellung ein: Die Angst ist eine unserer ersten Emotionen (wenn nicht sogar die erste), zu der wir fähig sind und bleibt unser ganzes Leben lang als anderen Gefühlen vorgestellte Emotion bestehen. In diesem Sinne ist die Angst eine primär primitive Emotion, die zwar ein minimales Bewusstsein voraussetzt, jedoch keine kausale Denkfähigkeit benötigt: "Die Angst, genetisch gesehen die erste unter den Emotionen, bleibt als Untergrund aller Gefühle vorhanden. Sie infiziert sie alle und nagt an den Rändern von Liebe und Gegenseitigkeit. ${ }^{17}$ Vereinfacht ausgedrückt entsteht die Angst in Situationen der Hilflosigkeit (der Säugling kann sich nicht selbst am Leben erhalten) und ist somit eine für das Überleben notwendige Emotion, denn sie ermöglicht es dem Säugling, andere dazu zu bringen, sich um ihn zu kümmern. ${ }^{18}$ Die Angst ist in ihren Grundzügen aber nicht nur primitiv, sie ist auch asozial. Denn auch wenn wir als Erwachsene durchaus in der Lage sind, um Andere zu fürchten, so ist die Angst meistens eine auf uns selbst gerichtete - und damit narzisstische - Emotion, die uns oftmals auf die Stufe eines kindlichen Solipsismus zurückwirft. ${ }^{19,20}$ Insofern kann die Angst also auch als infantil und damit als der Vernunft prima facie entgegengesetzte Emotion verstanden werden.

\footnotetext{
${ }^{16} \mathrm{Vgl}$. Aristoteles, Rhetorik II, in: Ders., Werke in deutscher Übersetzung, Bd. 4, Berlin 2002, 5,1382a 21-25.

${ }_{17}$ Nussbaum, Königreich der Angst, 39.

${ }^{18}$ Vgl. ebd., 41, 46.

${ }^{19}$ Einige ForscherInnen gehen sogar so weit, die Sorge um andere als narzisstische Sorge zu bezeichnen, da sich die Emotion zwar nicht zwingend auf einen selbst bezieht, aber doch stets auf das "größere Selbst « - auf Familie, Freunde usw. Die Anwendung unserer moralischen Fähigkeiten ist insofern also grundsätzlich narzisstisch.Vgl. ebd., 53. Siehe auch Donald W. Winnicott, Reifungsprozesse und fördernde Umwelt. Studien zur Theorie der emotionalen Entwicklung [1965], Gießen 2002; Ders.,Vom Spiel zur Kreativität [1971], Stuttgart 2006. Daniel Stern, The Interpersonal World of the Infant. A View from Psychoanalysis and Development Psychology, London 1985. ${ }^{20} \mathrm{Vgl}$. Nussbaum, Königreich der Angst, 49.
} 
Die Angst ist aber, so Nussbaum, nicht notwendigerweise unvernünftig oder sogar per se unbegründet, sobald wir unsere kindliche Abhängigkeit überwunden haben. Es kann durchaus vernünftig sein, sich als erwachsene Person vor oder um gewisse Dinge zu fürchten. Die Angst muss aber der skeptischen Prüfung standhalten, wir müssen also fähig sein, innezuhalten, um über unsere eigene Angst kritisch und reflektiert nachdenken zu können. Wir müssen uns vor Augen halten können, woher die Angst kommt, worauf sie sich bezieht und wohin sie uns führt. ${ }^{21}$ Nussbaum ist überzeugt, dass nur die vernünftige Angst gute Handlungsempfehlungen geben kann. Bleibt die Angst hingegen irrational - und damit auf der Stufe der primitiven, infantil-narzisstischen Urangst (Nussbaum nennt diese Angst auch "gestaltlos") -, bedroht sie laut Nussbaum das gegenseitige und gerechte Zusammenleben, denn ein solches setzt die Fähigkeit voraus, sich auch um jene ernsthaft zu sorgen, die nicht zum "größeren Selbst" gehören. Zudem schränkt die Angst unser rationales Denkvermögen ein, was den Irrtum begünstigt und es uns erheblich erschwert, die eigene Angst einer skeptischen Prüfung zu unterziehen. ${ }^{22}$ Wir müssen also unseren narzisstischen Solipsismus erfolgreich überwinden, damit ein demokratisch-selbstbestimmtes Zusammenleben möglich ist.

Laut Nussbaum ist das im Grunde leicht: Es muss uns nur gelingen, dasjenige, wovor wir uns fürchten, aus einem anderen Blickwinkel heraus zu betrachten, nämlich demjenigen der Hoffnung. Der Unterschied liegt also nicht im Gegenstand, worauf sich die jeweilige Emotion bezieht, sondern in der Perspektive, die wir auf diesen Gegenstand haben. Nussbaum schreibt:

Aber wenn ihnen [der Angst und der Hoffnung] im Prinzip die gleiche Idee zugrunde liegt - nämlich, dass der ersehnte Ausgang einer Sache ungewiss ist - und wenn es nicht Wahrscheinlichkeiten sind, die den Unterschied ausmachen: Worin besteht dann der Unterschied in den Gedanken und Einstellungen einer Person, der zu diesem Unterschied des Gefühls führt (oder von ihm begleitet wird)? Es scheint, als läge ein Unterschied im Schwerpunkt der Aufmerksamkeit vor, ähnlich wie bei einem Glas, das man als halb leer oder halb voll betrachten kann. Es handelt sich um dasselbe Glas, das unterschiedlich gesehen wird. Bei der Angst liegt der Schwerpunkt auf dem schlechten Aus-

${ }^{21}$ Vgl. ebd., 22.

${ }^{22}$ Vgl. ebd., 112. 
gang, der sich ergeben wird, bei der Hoffnung liegt er auf dem guten Ausgang. ${ }^{23}$

Die Vision einer besseren, möglichen Welt (und das bedeutet, dass diese Verbesserung durch unsere Handlungen vorangetrieben werden kann) ist ein zentrales Element von Nussbaums Vorschlag einer angstfreien, demokratischen Gesellschaft. Wir müssen erkennen, dass die Hoffnung genau dort, wo die infantile Angst lähmt, zur Handlungsfähigkeit antreiben kann (sofern es sich um praktische Hoffnung und nicht um untätige Hoffnung handelt) ${ }^{24}$ Im Grunde hängt es folglich von uns selbst ab, wie unser (politisches) Zusammenleben gestaltet ist. Wir entscheiden darüber, ob und wann wir unsere Angst überwinden, um eine demokratische - und damit gerechte, liebende und einander vertrauende - Gesellschaft zu ermöglichen: "Hoffnung ist eine Wahl, die man trifft, und sie ist eine praktische Gewohnheit. $\ll^{25}$

Es ist folglich die gestaltlose Angst, die Nussbaum als eines der Hauptprobleme unserer momentanen politischen Situation (und damit ist nicht nur, aber auch, die Wahl Donald Trumps als Präsident der USA gemeint ${ }^{26}$ ) identifiziert. So führt die durch Angst geschürte Wut auf Andere und Fremde zu einem gesteigerten Bedürfnis nach Sicherheit, das nicht nur eine wichtige Rolle für die Legitimierung autoritärer Politik- und PolitikerInnentypen spielt, die aktuell in zahlreichen westlichen Demokratien an Macht gewinnen. Sie stellt auch eine Bedrohung für jene politischen Institutionen und Verfahren der demokratischen Meinungsbildung dar, in denen die Werte der Pluralität und kollektiven Selbstbestimmung hochgehalten werden. ${ }^{27,28}$ Denn die gestaltlose Angst steht aufgrund ihrer oftmals unberechtigten und durch politische Rhetorik beeinflussten Selbstbezogenheit im Gegensatz zur demokratischen, selbstbestimmten Lebensführung, die ihrerseits eine Gemeinsamkeit in Gleichheit und Vertrauen ermöglicht: "Die Angst schwelt ständig unter der Oberfläche der moralischen Anteilnahme und droht, die Demokratie zu destabilisieren; denn die Demokratie verlangt von uns allen,

\footnotetext{
23 Ebd., 239.

24 Vgl. ebd., $240 f$.

25 Ebd., 245.

26 Vgl. ebd., 7-18.

27 Vgl. ebd., 7.

28 Vgl. Leonard Cruz/Steven Buser (Hg.), A Clear and Present Danger: Narcissism in the Era of President Trump, Nashville, NC 2016.
} 
dass wir unseren Narzissmus einschränken und die Gegenseitigkeit akzeptieren. ${ }^{29}$

Auch wenn diese Diagnose die gegenwärtigen politischen Transformationsprozesse im Kontext der sogenannten »autoritären Wende ${ }^{30}$ sicherlich in vielerlei Hinsicht treffend analysiert, so zeigt sich doch bei einer genaueren Inblicknahme anderer zeitgenössischer Bezugnahmen auf die Emotion der Angst, dass Angst keineswegs stets dieselbe, manifest oder latent demokratiegefährende Rolle spielt. Insbesondere die Bezugnahme auf das Motiv der Angst im Kontext der aktuellen Klimabewegung, namentlich in den Reden ihrer Vorreiterin Greta Thunberg, ist für unsere vorliegenden Überlegungen besonders interessant, insofern sie gerade einige bei Nussbaum entwickelten Thesen zum Verhältnis von Angst und Politik auf die Probe zu stellen vermag. Es ist Ziel des nächsten Abschnitts, die Funktion der Angst in Thunbergs Klimaaktivismus anhand von drei Aspekten aufzuschlüsseln, um davon ausgehend die Stärken und Schwächen der Nussbaum'schen Theorie sowie deren Erklärungspotential noch einmal kritisch zu beleuchten.

\section{Die Funktion von Angst im aktuellen Klimaakti- vismus}

Im Rahmen ihrer Rede anlässlich des Treffens des Weltwirtschaftsforums, das im Januar 2019 in Davos stattfand, rief Thunberg die anwesenden Politik- und WirtschaftsvertreterInnen dazu auf, angesichts der drohenden Klimakatastrophe in Panik zu geraten und sich in ihre eigene Angst einzufühlen: »I want you to panic, I want you to feel the fear I feel every day. And then I want you to act, I want you to act as if you would in a crisis. ${ }^{31}$ In der Tat artikuliert Thunbergs Rede eine tiefgreifende Erschütterung des Vertrauens in die etablierten politischen Entscheidungsinstanzen. Die Menschen wurden, so Thunberg, über das Ausmaß der Katastrophe zu lange im Dunkeln gelassen und mit der trügerischen Hoffnung auf

\footnotetext{
${ }^{29}$ Vgl. Nussbaum, Königreich der Angst, 84.

${ }^{30} \mathrm{Vgl}$. John Borneman, Border Regimes, the Circulation of Violence and the Neoauthoritarian Turn, in: A Companion to Border Studies, hg. v. Thomas M. Wilson/ Hastings Donnan, Oxford 2012, 119-135; Christian Kreuder-Sonnen, An authoritarian turn in Europe and European Studies?, in: Journal of European Public Policy 25/3 (2018), 452-464.

${ }^{31}$ https://www.fridaysforfuture.org/greta-speeches\#greta_speech_jan25_2019 (8.1.2020).
} 
technologische Innovationen vertröstet. Diesem Hoffnungsversprechen hält Thunberg die moralische Pflicht entgegen, angesichts der Klimakrise Verantwortung zu übernehmen und sofort zu handeln. Um die politischen und ökonomischen EntscheidungsträgerInnen von der Dringlichkeit dieser Pflicht zu überzeugen, rekurriert sie nicht zuletzt auf die Emotion der Angst - ihre Angst, die Angst ihrer Generation. Dabei fallt zudem auf, dass Thunberg Angst und Panik gerade nicht so versteht, dass politisches Handeln dadurch paralysiert wird. Vielmehr solle sich konzertiertes politisches Handeln gegen die Klimakatastrophe von der erschütternden Erfahrung der Angst und der Panik her verstehen (»and then I want you to act«). Angst hat für Thunberg also in erster Linie aktivierende und verbindende Funktion. Dies lässt sich konkret anhand von drei Aspekten veranschaulichen:

1. Erstens lässt sich beobachten, dass Thunbergs Bemühen von Angst dezidiert das Ziel der Einung verfolgt. Dieses Ziel verdeutlicht nicht zuletzt der Slogan »Unite behind the scientists«: Es soll eine weltweite, gemeinsame politische Agenda und Aktion geschaffen werden. Oder anders formuliert: Der Klimawandel wird in Thunbergs Aufruf I want you to panic! gerade politisiert. Er wird zu einer gemeinsamen Angelegenheit gemacht, die wir nicht verleugnen können und um die wir uns alle zu kümmern, zu streiten und zu deren Lösung wir gemeinsam beizutragen haben. Thunbergs AngstDiskurs zielt damit auf die Stiftung eines verbindenden, kollektiven "Wir«, das gemeinsam für umweltpolitische Agenden eintritt.

2. Der Aufruf, der Wissenschaft Gehör zu schenken, verweist auf einen zweiten zentralen Aspekt, der den Status bzw. die Anerkennung wissenschaftlicher Erkenntnisse (»Tell the truth!«) betrifft. So bezieht sich die Umweltbewegung auf Angst, um die zerstörerische Dimension des Klimawandels sichtbar und greifbar zu machen. Thunbergs Aufruf zur Panik zielt dabei darauf, Angst angesichts der Faktizität des Klimawandels zu vermitteln. Angst bleibt hier aber nicht unreflektiert, sondern ist gerade das Ergebnis eines Reflexionsprozesses: Wer um das Ausmaß der Klimakatastrophe Bescheid wisse, so Thunberg, gerate unweigerlich in Panik.

3. Ein dritter Aspekt betrifft die Funktion von Angst für die Mobilisierung politischerVeränderung und die Frage, welche Rolle dabei die Vision einer besseren Zukunft einnimmt. Die Ausrichtung hin auf eine bessere Zukunft wird anhand einer direkten Konfrontation zwischen Trump und Thunberg besonders anschaulich: So verspottete Trump Thunberg nach ihrer Rede am New Yorker UN-Klimagipfel in einem Tweet und nannte sie ironisch "a very happy young 
girl looking forward to a bright and wonderful future.$^{32}$ Thunbergs Reaktion auf Trump bestand darin, dass sie sich seine Zuschreibung postwendend für ihre Twitter-Biographie aneignete, dabei aber gerade den ironischen Gestus von Trumps Aussage zurückwies. ${ }^{33}$ Während Trump die Hoffnung auf eine bessere Zukunft lächerlich macht, bestätigt Thunberg dagegen: Ja, es geht ihr um die Zukunft.Vor dem Hintergrund dieser symbolischen Geste wird auch die Differenz zwischen den jeweiligen Bezugnahmen auf die Emotion der Angst deutlich: Während Trump seine Wähler mit der Angst vor der Zukunft zu bewegen sucht - vor dem Anderen und Fremden, vor dem Verlust des Status quo und der eigenen Privilegien -, mobilisiert Thunberg die Angst um die eigene Zukunft. Thunberg und ihren MitstreiterInnen geht es darum, über ihre Zukunft selbst mitzubestimmen. Dafür rufen sie nicht zuletzt zu einer Öffnung gegenüber gesellschaftlichen Alternativen auf (auf den Punkt gebracht mit dem Slogan: »Systemwandel statt Klimawandel«).

Anhand dieser drei Beobachtungen lassen sich nun auch konkrete Rückfragen an Nussbaums Überlegungen zur politischen Rolle von Angst formulieren:

Ersten lässt sich fragen, wie aus Nussbaum'scher Perspektive der Umstand bedacht werden kann, dass Angst offenbar nicht nur eine spaltende, sondern auch eine verbindende Qualität zukommt. Denn Thunbergs Aktivismus soll ja nicht zu Spaltung führen, sondern vielmehr eine solche verhindern: Gemeinsam können wir es schaffen, dem Klimawandel Einhalt zu gebieten.Vor diesem Hintergrund geht es genau darum, dass die Sorge um das Klima keine bloß individuellsubjektive Angelegenheit bleibt, die die Einzelnen für sich affiziert, sondern gerade als kollektive Sorge oder Angst zum Einsatzpunkt gemeinsamer politischer Aktion wird. Auch wenn sich Nussbaum insgesamt von der Angst als gerechtfertigtes, politisches Movens distanziert, ${ }^{34}$ so gesteht sie ihr (oder vielmehr dem Zorn, der eng mit der Angst verknüpft ist) dennoch in seltenen Umständen ein gewisses Potential zu: »Ich werde geltend machen, dass der Aspekt der Empörung persönlich und gesellschaftlich wertvoll ist, wenn un-

\footnotetext{
32 https://www.theguardian.com/us-news/2019/sep/24/she-seems-very-happytrump-appears-to-mock-greta-thunbergs-emotional-speech (8.1.2020).

${ }^{33}$ https://www.spiegel.de/lebenundlernen/schule/greta-thunberg-trollt-trumpmit-twitter-bio-a-1288470.html (8.1.2020).

${ }^{34}$ Vor allem distanziert sich Nussbaum auch von der weit verbreiteten Idee, der Zorn (der laut Nussbaum auf Angst basiert) sei notwendig dafür, die eigene Würde und Selbstachtung zu wahren, oder gar essentiell für den Kampf gegen Ungerechtigkeit. Vgl. Martha C. Nussbaum, Anger and Forgiveness, Resentment, Generosity, Justice, Oxford 2016, 6 .
} 
sere Überzeugungen der Wahrheit entsprechen. ${ }^{35}$ Diese Empörung definiert Nussbaum als Zorn des Übergangs. Ähnlich wie der Zorn hat er einen Schaden zum Gegenstand, der auf ungerechtfertigte Weise zugefügt wurde. Ziel des Zorns des Übergangs ist es im Gegensatz zum herkömmlichen Zorn allerdings nicht, Vergeltung üben zu wollen, sondern auf konstruktive Weise den Schaden zu beheben. ${ }^{36}$ Insofern muss Thunbergs Empörung und ihr damit einhergehender Rekurs auf die Angst innerhalb der Nussbaum'schen Theorie der Emotionen als Zorn des Übergangs verstanden werden, und nicht als narzisstisch-ausgrenzende Angst. ${ }^{37}$ Allerdings kann auch der Zorn des Übergangs - trotz seiner gerechtfertigten Funktion als Protestmittel - nur als Zwischenstadium auf dem Weg zu einer besseren Zukunft dienen. Denn Nussbaums Theorie der Angst geht mit einer Hierarchisierung der Emotionen einher, in welcher der infantilen Angst eine verursachende und andere Emotionen begründende Rolle zukommt. Viele andere, die demokratische Selbstbestimmtheit gefährdende Emotionen (wie bspw. Ekel oder Neid) setzen die infantile Angst notwendigerweise voraus. So auch der Zorn des Übergangs. Schließlich muss auch er zugunsten der Hoffnung überwunden werden. ${ }^{38}$

Zweitens lässt sich fragen, wie mit Nussbaum der Beobachtung Rechnung getragen werden kann, dass KlimaaktivistInnen auf eine Angst rekurrieren, die das Ergebnis eines Prozesses der Reflexion und der Anerkennung wissenschaftlicher Erkenntnisse darstellt. Vor diesem Hintergrund hält Thunbergs Angst dem Nussbaum'schen Anspruch, einen rationalen Reflexionsprozess zu durchlaufen, durchaus stand, und ist insofern weder gestaltlos noch narzisstisch, sondern klar umrissen und begründet - und damit nicht per se negativ zu bewerten. Auch diese Beobachtung spricht dafür, Thunbergs tragende Emotion eher als Zorn des Übergangs denn als Angst zu begreifen. Mit Blick auf diese Überlegungen erscheint es produktiv, ausgehend von Nussbaum den Übergang von Angst bzw. Zorn des

\footnotetext{
${ }^{35}$ Nussbaum, Königreich der Angst, 98.

${ }^{36}$ Vgl. ebd., 115; Nussbaum, Anger and Forgiveness, 35f.

${ }^{37}$ Genau genommen muss hier eine Unterscheidung gemacht werden zwischen Thunbergs Emotion - dem Zorn des Übergangs - und der Emotion, die sie bei anderen auslöst. Nussbaums Theorie der Emotionen und ihre Angstkonzeption legen nahe, dass die eigentliche Problematik in dieser Übertragung angesiedelt ist. Es ist anzunehmen, dass auch der Zorn des Übergangs vorerst (infantile) Angst und Zorn auslöst, der Vergeltung will. Der Zorn des Übergangs lässt sich nicht übertragen. Es liegt in derVerantwortung der Einzelnen, sich auf geeignete Weise mit ihren eigenen Emotionen auseinanderzusetzen.

${ }^{38}$ Vgl. Nussbaum, Königreich der Angst, 111.
} 
Übergangs zu Hoffnung noch einmal genauer in den Blick zu nehmen. Unklar bleibt nämlich, ob die Reflexion von Angst für Nussbaum dazu führt, dass sie zugunsten von Hoffnung überwunden wird, oder ob die Reflexion einen Transformationsprozess - der aber keiner gänzlichen Tilgung der Angst gleichkommt - hin zur Emotion der Hoffnung eröffnet. Mit der zweiten Lesart könnte man Nussbaums Theorie dann so verstehen, dass der Perspektivenwechsel (von Angst zur Hoffnung) nur dann stattfinden kann, wenn die zunächst infantile Angst einer begründeten Angst weicht und sich der Perspektivenwechsel somit auf die begründete Angst bezieht. Denn der eigentliche Unterschied liegt ja in der (vernünftigen) Betrachtung des Gegenstands; die infantile Angst hingegen ist solchen Betrachtungen vorgeordnet. Das Phänomen der Angst bei Thunberg ließe sich damit auf jeden Fall angemessener erklären. Gerade weil sich Thunberg der Angst keineswegs als bloßer Panikmacherei bedient, sondern mit dieser Angst gerade eine positiv konnotierte Zukunftsvision transportiert, ist es durchaus plausibel, sie im Nussbaum'schen Sinne in erster Linie als Hoffnungsträgerin zu verstehen. 


\section{Ausblick}

Mit Nussbaums Theorie der Emotionen und ihrer Theorie der Angst im Besonderen eröffnen sich Möglichkeiten, das Phänomen der Angst im Raum des Politischen nicht nur anzuerkennen, sondern auch erneut zu besprechen. Gegen eine weit verbreitete und im politischen Liberalismus ausformulierte Vorstellung des Politischen als eines Kooperationsraumes zwischen rationalen Akteuren, in dem Emotionen keine Rolle spielen (dürfen), stellt sie heraus, dass Emotionen nicht nur notwendigerweise Bestandteil der politischen Sphäre sind; vielmehr möchte Nussbaum auch zeigen, dass es wichtig und sinnvoll ist, mit Emotionen zu arbeiten, um Gerechtigkeit zu erwirken. ${ }^{39}$ Insgesamt hält sie diese Perspektive auch in ihren Überlegungen zur Angst aufrecht. Dies ist ein wichtiges Ergebnis unserer Ausführungen, denn damit gesteht sie selbst dem so ursprünglichen Gefühl der Angst eine Gestaltbarkeit und eine Funktion zu.

Ist die Diagnose jedoch richtig, dass Angst nicht nur ein randständiges Gefühl, sondern ein für die heutige Verständigung über das Politische sehr zentrales Thema ist, dann ist nicht mehr ganz so deutlich, was ihre Theorie ausrichten kann. Angst scheint weder ein singuläres Gefühl zu sein, das die Einzelnen empfinden; noch steht Angst in Isolation - vielmehr geht es im Politischen um eine Gemengelage von Angsteinflößung und Macht, von Instrumentalisierung von Angst, und von Angst in einem Feld von Wut, Hass und anderen archaisch anmutenden Affekten. Zugespitzt formuliert möchte man bezweifeln, dass sich Einzelne daraus befreien können, indem sie schlichtweg anders denken oder Angst durch Hoffnung ersetzen. Angst ist zu sehr verstrickt in Mechanismen der Macht, der zunehmenden Vereinzelung und auch der tatsächlichen Hoffnungslosigkeit, als dass ein so schlichtes Mittel der Auflösung überzeugen könnte.

Es wäre also wünschenswert, den Übergang von der primitiven Angst hin zu einer Politik der Hoffnung umfangreicher auszugestalten und Kriterien festzumachen, die diesen Übergang für möglichst alle Bevölkerungsschichten zugänglich machen, auch unter prekären Umständen und in Situationen von begründeter, akuter Angst um die eigene Existenz. Die von Nussbaum vorgebrachten Vorschläge sind sicherlich ein guter Anfang. Zentrale Aspekte einer Politik der Hoffnung sind ihrer Meinung nach die geteilte Liebe, das Erschaffen von phantasievollen Bildern der Zukunft durch Musik, Gedichte und andere Kunstformen, und die Fähigkeit zur Reflexion sowie zur

${ }^{39}$ Vgl. Nussbaum, Political Emotions. 
vernünftigen Kritik. ${ }^{40}$ Problematisch bleibt, dass Nussbaum außerordentlich hohe Ansprüche an die Fähigkeit zur rationalen Reflexion und der vernunftgeleiteten Selbstgestaltung und -zügelung der Einzelnen stellt. Auch mutet ihre Politik der Hoffnung bisweilen gar elitär an - inwiefern etwa die Lektüre von Shakespeares Hamlet und sonstige kulturelle Tätigkeiten unsere politische Lage verbessern, sei dahingestellt. Dennoch, und das möchten wir zum Schluss noch einmal besonders hervorheben, leistet Nussbaum mit ihrer Theorie der Angst einen wichtigen Beitrag zum Diskurs um die Angst in der politischen Philosophie. Denn es gelingt ihr - wie am Beispiel von Greta Thunberg veranschaulicht - auch jene Phänomene einzufangen und zu erklären, die in der gängigen Angstrhetorik nur schlecht ihren Platz finden.

- Eva Bobst ist wissenschaftliche Mitarbeiterin am Institut für Philosophie an der Universität Wien. Ihre Forschungsschwerpunkte liegen im Bereich der Ethik und der angewandten Ethik. Aktuell arbeitet sie zu Fragen der ethischen Entscheidungsfindung und der weisen Lebensführung.

- Prof. Dr. Angela Kallhoff ist Universitätsprofessorin für Ethik mit besonderer Berücksichtigung für Angewandte Ethik an der Universität Wien. Sie arbeitet zu Themen der Ethik, der angewandten Ethik und politischen Philosophie.

- Anna Wieder ist wissenschaftliche Mitarbeiterin am Institut für Philosophie der Universität Wien. Ihre Forschungsschwerpunkte liegen im Bereich der Ethik und der Politischen Philosophie, insbesondere der Demokratietheorie. Aktuell arbeitet sie zu Phänomenen und Konzepten des politischen Widerstands und Protests.

${ }^{40}$ Vgl. Nussbaum, Königreich der Angst, 235. 